\title{
SAND TRANSPORT BY SURFACE WAVES: CAN STREAMING EXPLAIN THE ONSHORE TRANSPORT?
}

\author{
Wouter Kranenburg ${ }^{1}$, Jan Ribberink ${ }^{1}$ and Rob Uittenbogaard ${ }^{2}$
}

\begin{abstract}
In wave flumes an onshore boundary layer current is present that is not present in oscillating flow tunnels. We investigate numerically the hypothesis that this streaming explains the measured increase of onshore directed sediment transport in flumes over tunnels. In the formulation and validation of the model special attention has been given to the wave-generated net current profile. From model experiments we conclude that the additional current indeed contributes to onshore transport, but can not be the full explanation of the measured differences in transport rates. Other contributing mechanisms are the amplification/reduction of the fall velocity by vertical sediment advection (only relevant for fine grains) and the amplification/reduction of the concentration at maximum onshore/offshore velocity by intra-wave gradients in horizontal sediment flux. The latter contributes, for the investigated cases, to onshore transport with comparable order as the boundary layer current. These conclusions are relevant for further development of parameterizations of wave-induced sediment transport for morphodynamic models.
\end{abstract}

Keywords: sediment transport, waves, boundary layers, streaming, sheet-flow

\section{INTRODUCTION}

We study sediment transport under waves, focusing on the effects of wave-induced boundary layer streaming. This streaming is a net current close to the bed, generated by the combination of boundary layer turbulence and vertical orbital motions. We are especially interested in the influence of streaming on sediment transport in the sheet-flow regime. In that transport regime, occurring under highly energetic but non-breaking waves, bedforms are washed away and a layer of highly concentrated sediment is set in motion, which can lead to high transport rates.

Sediment transport determines the development of bed morphology. Good predictions of morphological changes, e.g. of cross-shore coastal profiles, are essential for coastal safety and coastal management. Morphological changes usually take place on a timescale much longer than the wave period. But because such changes are determined by the sediment transport, it is vital to achieve a good understanding of the small-scale processes that determine the sediment transport and to develop ways to quantify their effects.

Earlier research on sediment transport under waves has indentified several important transport mechanisms. We mention the effects of nonlinear wave shapes, boundary layer streaming phenomena, breaking-induced undertow currents and slope effects.

Nonlinear wave shapes develop during the propagation of a wave towards the shore. The velocity near the bed changes with the wave shape and can be characterized in terms of velocity skewness and acceleration skewness (Elgar and Guza, 1985). These phenomena have been thoroughly studied in isolation in oscillating flow tunnels. In the case of velocity skewness (example: 2nd order Stokes waves), onshore velocity peaks are both high and short, while the offshore velocity is smaller, but lasts longer. The grain size determines whether this will result in onshore or offshore transport. Fine grains are readily brought into suspension, but settle slowly. Therefore, in contrast to medium grains, fine sediments can still be present in the flow in considerable amounts at the moment the flow reverses from onshore to offshore (phase-lag effect, Dohmen-Janssen et al., 1999, 2002b). This can result in net offshore sediment transport for fine grains in oscillating flow tunnels. In the case of acceleration skewness (steep wave front), the moment of maximum onshore bed shear stress comes early in the onshore phase of the horizontal velocity, while the moment of maximum offshore bed shear stress displaces towards the end of the offshore phase of the horizontal velocity. This leads to onshore sediment transport for both fine and medium grain sizes.

Boundary layer streaming denotes the generation of a net current close to the bed. We distinguish between asymmetry-induced streaming and real-wave-induced streaming. Streaming of the first type is related to the time variation of turbulence under unsteady flow (Trowbridge and Madsen, 1984). In the

\footnotetext{
${ }^{1}$ Water Engineering and Management, University of Twente, Box 217, 7500 AE, Enschede, The Netherlands; w.m.kranenburg@utwente.nl; j.s.ribberink@utwente.nl; 
case of asymmetry in velocity in successive wave half cycles, the (time dependent) turbulence intensities will also show asymmetry, resulting in non-zero wave-averaged Reynolds stresses. For flat beds, this causes a net offshore current close to the bed. Such currents have been observed by Ribberink and Al-Salam (1995) in oscillating flow tunnel (U-tube) experiments and reproduced by Davies and Li (1997) using a numerical boundary layer model. In contrast to the situation in merely oscillating flow, the velocity field under real waves is horizontally non-homogeneous. The horizontal gradients and their associated vertical (orbital) velocities produce the second type of boundary layer streaming (Longuet-Higgins, 1953/1958). Boundary layer turbulence affects the phase of the velocities such that horizontal $\left(\begin{array}{l}0 \\ u\end{array}\right)$ and vertical ( $\underset{w}{w}$ ) velocity components are not entirely out of phase (as would be the case in irrotational flow). This results in a non-zero wave-averaged stress term that causes a net current close to the bed in onshore direction. Note that this type of streaming is also present under sinusoidal waves.

The expectation that real-wave-induced boundary layer streaming contributes to onshore sediment transport has been the motive for experimental research in full-scale wave flume facillities (DohmenJanssen and Hanes, 2002a, 2005, Schretlen, 2010). Indeed onshore transport has been observed, both for medium and small grain sizes. However, unlike with wave shape aspects, streaming influence can not be studied in isolation. The non-homogeneous velocity field under real waves not only induces streaming, but simultaneously generates onshore mass transport by Stokes' drift. Consequently, also a return current will be generated, because closed flumes force the wave-averaged mass transport to be zero. All these real-wave effects influence the net current profile. Furthermore, the intra-wave horizontal gradients in velocity and concentration and the vertical velocities might also have a direct influence on advective transport of sediment.

To answer the question whether the real-wave-induced streaming is indeed the explanation for the differences in sediment transport rates between tunnels and flumes, we deploy a numerical model, which gives the possibility to investigate processes in isolation and to quantify their contribution to sediment transport. Most numerical models presently used for reproduction and interpretation of Utube, flume and prototype measurements on sediment transport are first or second order boundary layer models. Examples of applications, e.g. in the reproduction of asymmetry-induced streaming, wave shape effects and even the morphological development of a cross-shore profile (bar migration), are Hoefel and Elgar (2003), Henderson et al. (2004), Hassan and Ribberink (2010), Ruessink et al. (2009), Holmedal and Myrhaug (2009). These boundary layer models are forced by a horizontal pressure gradient or a horizontal velocity at the edge of the wave boundary layer (for most models the top of their domain). In first order BL models real-wave-induced streaming could only be accounted for by adding a measured or estimated (e.g. using Longuet-Higgins' expression) contribution to the forcing, while second order BL models in principle can compute streaming within the wave boundary layer, but miss the Stokes' drift and return current. For a good interpretation of the differences between tunnel and flume, we need to solve sediment transport under waves without a priori description of streaming velocities and with inclusion of Stokes' drift and return current.

In this study, we investigate the influence of streaming on sediment transport with a model that explicitly computes the wave-induced currents over the entire vertical profile (description in section 2). After validation of the hydrodynamics on the measured net current profiles, we include sediment in the simulations and compare the model predictions with data on transport rates in tunnels and flumes, the latter obtained during the full-scale experiments performed by Dohmen-Janssen and Hanes (2002) and Schretlen (2010). Subsequently, we study the distinct effects of real-wave-induced boundary layer streaming and other processes with numerical experiments (section 4). Our conclusions are summarized in Section 5.

\section{MODEL DESCRIPTION AND VALIDATION}

We investigate the sediment transport under surface waves with a 1DV-RANS model that solves equations for hydrodynamic pressure, orbital and wave-averaged velocity, turbulence properties and sediment concentration. This non-hydrostatic model has been developed originally to simulate wavecurrent interaction by Uittenbogaard (2000), has been applied on sediment transport by Bosboom \& Klopman (2000) and has been improved in its treatment of non-linear wave shapes during this study. 


\section{Assumption}

A basic premise is the flat bed assumption: strong changes of wave properties (height, shape) and wave-averaged velocities during propagation as well as bed forms (absent in the sheet-flow regime) are excluded from the modeling. As a result we can consider a wave of arbitrary shape as a sum of steady harmonic oscillations all propagating with the celeraty $c_{\mathrm{p}}$ of the carrier wave (bound waves). For each component we can relate (intra-wave) spatial and temporal derivatives with:

$$
\frac{\partial . .}{\partial x}=-\frac{1}{c_{p, x}} \frac{\partial . .}{\partial t} ; \quad \frac{\partial . .}{\partial y}=-\frac{1}{c_{p, y}} \frac{\partial . .}{\partial t}
$$

which makes it possible to compute the velocity field with a 1DV approach and to treat the (nonhydrostatic) orbital velocity components and (hydrostatic) wave-averaged velocity seperately.

\section{Solution procedure}

The non-linear Reynolds' averaged horizontal momentum equations describing the combined flow of waves and current are:

$$
\begin{aligned}
& \frac{\partial u}{\partial t}+u \frac{\partial u}{\partial x}+v \frac{\partial u}{\partial y}+w \frac{\partial u}{\partial z}=-\frac{1}{\rho} \frac{\partial p}{\partial x}-\left\{\frac{\partial \overline{u^{\prime} u^{\prime}}}{\partial x}\right\}-\left\{\frac{\partial \overline{u^{\prime} v^{\prime}}}{\partial y}\right\}-\left\{\frac{\partial \overline{u^{\prime} w^{\prime}}}{\partial z}\right\} \\
& \frac{\partial v}{\partial t}+u \frac{\partial v}{\partial x}+v \frac{\partial v}{\partial y}+w \frac{\partial v}{\partial z}=-\frac{1}{\rho} \frac{\partial p}{\partial y}-\left\{\frac{\partial \overline{v^{\prime} u^{\prime}}}{\partial x}\right\}-\left\{\frac{\partial \overline{v^{\prime} v^{\prime}}}{\partial y}\right\}-\left\{\frac{\partial \overline{v^{\prime} w^{\prime}}}{\partial z}\right\}
\end{aligned}
$$

Using the consequences of the flat bed assumption, intra-wave and wave-averaged velocities are solved along the following computation procedure (schematically illustrated in Figure 1): Per spectral component, the hydrodynamic pressure $\not{p}$ is solved from a linearized Poisson equation. Consequently, the vertical orbital velocity $\underset{w}{w}$ is solved from a linearized vertical orbital momentum equation. With the obtained $\not{p}$ and $\stackrel{w}{w}$, we solve $\stackrel{\circ}{u}$ from a non-linear Reynolds-averaged horizontal orbital momentum equation with turbulence expressed in velocity gradients using the Boussinesq hypothesis. Each time step, this loop (1) is carried out for each wave component. Subsequently, the sum of the velocities is used to compute the rate of change of turbulence properties with a $k$ - $\varepsilon$ turbulence model. Total velocities and turbulence quantities are used to determine the sediment transport (loop 2). After completion of a wave period, wave-averaged quantities are computed for each component. In stead of literary computing the average of each non-linear term, we determine how large the sum of these terms should be to keep the average of the horizontal orbital velocity $\bar{u}=0$. This wave-averaged contribution (wave-current interaction force $F_{\text {wci }}$ ) is the coupling between the orbital and wave-averaged momentum equation.

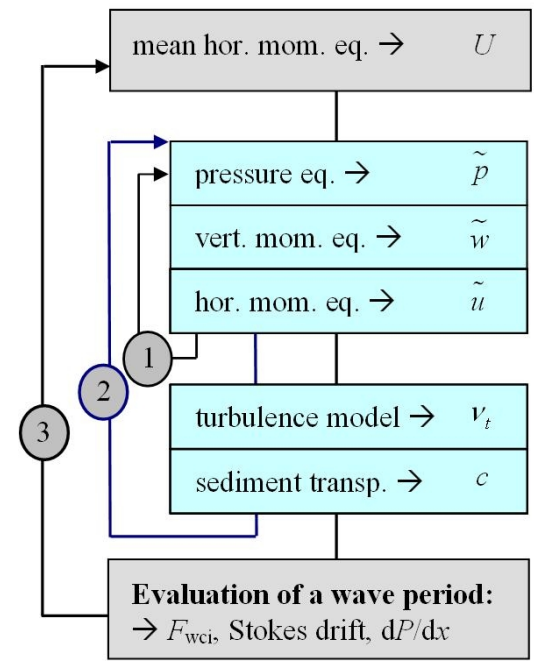

Figure 1: Diagram of the model structure 
Subsequently, we compute the (wave-averaged) Stokes drift and the mean horizontal pressure gradient. The latter depends on the requirement that the mass transport in a closed flume averaged over the wave period and depth should be zero. Finally, we compute the wave-averaged velocity $U(z)$ (loop 3 ) and make use of the result for computation of orbital velocities, turbulence quantities and sediment transport in the next wave period. The equations are solved through finite difference discretization on a non-equidistant vertical grid, which gives the possibility to resolve the wave boundary layer in detail while simulating the flow along the entire water depth without too much computational effort.

\section{Model validation (hydrodynamics)}

The hydrodynamics of the model has been validated by comparison with flume experiments of Klopman (1994). We compare the predicted mean current profile and the predicted amplitude of the first harmonic of the horizontal velocity with measurements for a mono-chromatic wave with period $1.44 \mathrm{~s}$ and amplitude $0.06 \mathrm{~m}$ in a flume with water depth $0.5 \mathrm{~m}$ (Figure 2). Close to the bed the measured profile shows a distinct net current in the direction of wave propagation. This is the real wave induced streaming. Higher up in the vertical the mean velocity is negative, which is the effect of the return current that compensates mass transport in positive direction. The first harmonic shows a velocity overshoot in the boundary layer and a gradual increase higher up in the vertical. All these characteristics are reproduced very well by the model both qualitatively and quantitatively, where only the backward bending of the mean velocity around $0.5 \mathrm{~cm}$ could be considered as a slight deviation. This comparison shows that the model is able to predict the net current and intra-wave velocities under surface waves.
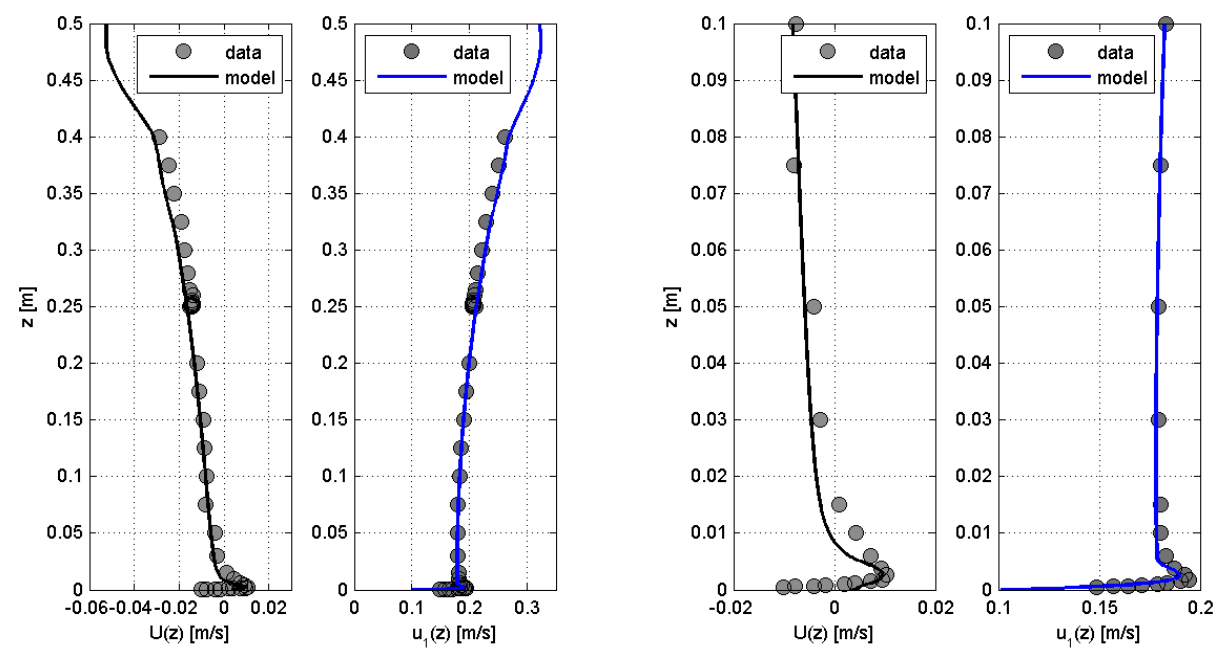

Figure 2: Profile of mean velocity $U(z)$ and amplitude of first harmonic of the horizontal velocity $u_{1}(z)$ for a mono-chromatic wave with period $T=1.44 \mathrm{~s}$ and amplitude $a=0.06 \mathrm{~m}$ in a flume of water depth $h=0.5 \mathrm{~m}$. Left: profile for entire water depth. Right: lower $0.1 \mathrm{~m}$.

\section{Sediment dynamics}

The transport of sediment is included into the Reynolds-averaged model with an advectiondiffusion equation. We model the upward transport by turbulent diffusion with a formulation similar to the Boussinesq hypothesis using a sediment diffusivity $\varepsilon$ and neglect the horizontal diffusive exchange:

$$
\left.\frac{\partial c}{\partial t}+\nabla \Phi \underline{u c}\right)=\frac{\partial}{\partial z}\left(\varepsilon \frac{\partial c}{\partial z}\right) \quad ; \quad \varepsilon=v+\frac{v_{t}}{\sigma_{t}}
$$

where $v_{\mathrm{t}}$ is the turbulent eddy viscosity and $\sigma_{\mathrm{t}}$ is the Prandtl-Schmidt number. The advective velocities in horizontal direction contain mean and orbital velocities, while the vertical advective velocity is the sum of the fluid velocity and the (downward) fall velocity of the grains. For the latter we use the expressions of Van Rijn (1993) and take into account the reduction of the fall velocity by the presence of other grains using the formulation of Richardson and Zaki (1954). The $k-\varepsilon$ turbulence model contains buoyancy flux terms to account for the conversion of turbulent kinetic energy into potential energy that takes place when sediment is brought upward by vertical mixing. As bottom boundary condition of the sediment balance we use the pick-up formulation of Zyserman and Fredsøe (1994), prescribed at the reference level of $2 * d_{50}$. 


\section{Tunnel version}

The non-hydrostatic model described above is aimed to solve wave-averaged and intra-wave velocities over the entire water depth under a surface wave like in a wave flume. The horizontally uniform situation in an oscillating flow tunnel can be simulated with the hydrostatic tunnel version of the model. The latter is actually a first order boundary layer model and is the version that has been applied by Hassan and Ribberink (2010) and Ruessink et al. (2009) to simulate sediment transport in oscillating flow tunnels, using identical formulations for sediment dynamics.

\section{RESULTS FOR SEDIMENT TRANSPORT IN FLUME AND TUNNEL}

\section{Comparison of measurements and simulations}

We apply the model as described in the previous section, including the sediment module, to simulate the experiments on sediment transport under waves as carried out by Dohmen-Janssen (2002a) and more recently Schretlen (2010) in the Large Wave Flume (GWK) in Hannover. The tunnel version is used to simulate a series of tunnel experiments, namely O'Donoghue and Wright (2004 a,b), Wright and O'Donoghue (2002), Ribberink \& Chen (1993) and Ribberink and Al-Salem (1994, 1995). The conditions of the various experiments are given in Table 1, which is actually an excerpt from the SANTOSS database (see Van der Werf et al, 2009).

\begin{tabular}{|c|c|c|c|c|c|c|c|c|c|c|c|c|}
\hline $\mathrm{nr}$ & reference & code & $\begin{array}{l}\mathrm{Hw} \\
(\mathrm{m})\end{array}$ & $\begin{array}{c}T \\
(\mathrm{~s})\end{array}$ & $\begin{array}{c}\mathrm{d} 50 \\
(\mathrm{~mm})\end{array}$ & $\begin{array}{c}\text { uon } \\
(\mathrm{cm} / \mathrm{s})\end{array}$ & $\begin{array}{c}\text { uoff } \\
(\mathrm{cm} / \mathrm{s})\end{array}$ & $\mathrm{R}$ & $B$ & $\begin{array}{l}\text { Wave } \\
\text { shape }\end{array}$ & $\begin{array}{c}\text { unet } \\
(\mathrm{cm} / \mathrm{s})\end{array}$ & $\begin{array}{l}\text { zref } \\
(\mathrm{cm})\end{array}$ \\
\hline 1 & DOH2002 & $\mathrm{MI}$ & 1.35 & 6.5 & 0.240 & 102.50 & 74.50 & 0.579 & 0.502 & $\mathrm{ctr}$ & -4.50 & 10.3 \\
\hline 2 & DOH2002 & MH & 1.60 & 6.5 & 0.240 & 112.70 & 68.30 & 0.623 & 0.459 & ctr & -3.70 & 10.9 \\
\hline 3 & DOH2O02 & MF & 1.30 & 9.1 & 0.240 & 134.70 & 66.30 & 0.670 & 0.564 & ctr & -3.70 & 9.9 \\
\hline 4 & DOH2OO2 & ME & 1.50 & 9.1 & 0.240 & 150.20 & 58.80 & 0.719 & 0.562 & $\mathrm{ctr}$ & -5.20 & 9.9 \\
\hline 5 & SCH(ip) & Re1565_08F & 1.50 & 6.5 & 0.138 & 155.23 & 83.22 & 0.651 & 0.5 & ctr & -5.79 & 4.0 \\
\hline 6 & $\mathrm{SCH}$ (ip) & Re1265_08F & 1.20 & 6.5 & 0.138 & 124.84 & 74.81 & 0.625 & 0.5 & ctr & -2.95 & 4.0 \\
\hline 7 & $\mathrm{SCH}(\mathrm{ip})$ & Re1065_08F & 1.00 & 6.5 & 0.138 & 112.59 & 74.26 & 0.603 & 0.5 & $\mathrm{ctr}$ & -1.67 & 4.0 \\
\hline 8 & $\mathrm{SCH}(\mathrm{ip})$ & Re1575_08F & 1.50 & 7.5 & 0.138 & 170.32 & 69.14 & 0.711 & 0.5 & $\mathrm{ctr}$ & -9.15 & 4.0 \\
\hline 9 & $\mathrm{SCH}$ (ip) & Re1550_08F & 1.50 & 5.0 & 0.138 & 127.80 & 101.96 & 0.556 & 0.5 & ctr & -2.76 & 4.0 \\
\hline 10 & $\mathrm{SCH}$ (ip) & Re1565_07M & 1.50 & 6.5 & 0.245 & 166.46 & 91.59 & 0.645 & 0.5 & ctr & -2.70 & 4.0 \\
\hline 14 & $\mathrm{SCH}(\mathrm{ip})$ & Re1575_08M & 1.50 & 7.5 & 0.245 & 142.93 & 60.93 & 0.701 & 0.5 & ctr & -7.61 & 4.0 \\
\hline 15 & SCH(ip) & Re1565_08M & 1.50 & 6.5 & 0.245 & 157.64 & 89.64 & 0.638 & 0.5 & ctr & -5.94 & 4.0 \\
\hline 16 & $\mathrm{SCH}(\mathrm{ip})$ & Re1550_08M & 1.50 & 5.0 & 0.245 & 149.15 & 121.21 & 0.552 & 0.5 & ctr & -4.18 & 4.0 \\
\hline 17 & $\mathrm{SCH}(\mathrm{ip})$ & Re1265_08M & 1.20 & 6.5 & 0.245 & 151.54 & 88.51 & 0.631 & 0.5 & $\mathrm{ctr}$ & -3.44 & 3.0 \\
\hline 18 & ODO2004 & FA5010 & not rel. & 5.0 & 0.130 & 138.00 & 91.00 & 0.603 & 0.500 & sos & 3.00 & 8.0 \\
\hline 19 & ODO2004 & FA7515 & not rel. & 7.5 & 0.130 & 144.00 & 94.00 & 0.605 & 0.500 & sos & 0.00 & 8.0 \\
\hline 20 & WRI2002 & LA406 & not rel. & 4.0 & 0.130 & 120.70 & 70.90 & 0.630 & 0.500 & sos & $\mathrm{NaN}$ & $\mathrm{NaN}$ \\
\hline 21 & WRI2002 & LA612 & not rel. & 6.0 & 0.130 & 155.29 & 91.11 & 0.630 & 0.500 & sos & $\mathrm{NaN}$ & $\mathrm{NaN}$ \\
\hline 22 & RIB1993 & D11 & not rel. & 6.5 & 0.128 & 98.10 & 60.90 & 0.617 & 0.500 & sos & 1.90 & 25.0 \\
\hline 23 & RIB1993 & D12 & not rel. & 6.5 & 0.128 & 153.80 & 100.20 & 0.606 & 0.500 & sos & 6.20 & 25.0 \\
\hline 24 & RIB1993 & D13 & not rel. & 6.5 & 0.128 & 125.90 & 80.10 & 0.611 & 0.500 & sos & 4.10 & 25.0 \\
\hline 25 & RIB1993 & D14 & not rel. & 6.5 & 0.128 & 77.30 & 49.70 & 0.609 & 0.500 & sos & 1.70 & 25.0 \\
\hline 26 & RIB1994 & B1 & not rel. & 6.5 & 0.210 & 119.20 & 73.80 & 0.618 & 0.500 & sos & 0.80 & 20.0 \\
\hline 27 & RIB1994 & B3 & not rel. & 6.5 & 0.210 & 109.60 & 71.60 & 0.605 & 0.500 & sos & -1.60 & 20.0 \\
\hline 28 & RIB1994 & B7 & not rel. & 6.5 & 0.210 & 90.20 & 54.80 & 0.622 & 0.500 & sos & 4.80 & 20.0 \\
\hline 29 & RIB1994 & B8 & not rel. & 6.5 & 0.210 & 127.20 & 73.80 & 0.633 & 0.500 & sos & 3.80 & 20.0 \\
\hline 30 & RIB1994 & B13 & not rel. & 6.5 & 0.210 & 119.00 & 91.00 & 0.567 & 0.500 & sos & 1.00 & 20.0 \\
\hline 31 & RIB1994 & B17 & not rel. & 6.5 & 0.210 & 43.38 & 28.62 & 0.603 & 0.500 & sos & 0.62 & 20.0 \\
\hline 32 & RIB1994 & B18 & not rel. & 6.5 & 0.210 & 63.49 & 37.51 & 0.629 & 0.500 & sos & 0.51 & 20.0 \\
\hline
\end{tabular}


With:

\begin{tabular}{|c|c|}
\hline (ip) & in preparation \\
\hline$H_{\mathrm{w}}$ & wave height \\
\hline$T$ & wave period \\
\hline$D_{50}$ & median grain diameter \\
\hline$U_{\text {on }}$ & onshore orbital velocity peak \\
\hline$U_{\text {off }}$ & offshore orbital velocity peak \\
\hline$R$ & degree of orbital flow velocity skewness, $R=U_{\mathrm{on}} /\left(U_{\mathrm{on}}+U_{\text {off }}\right)$ \\
\hline$B$ & $\begin{array}{l}\text { degree of flow acceleration skewness, } B=a_{\max } /\left(a_{\max }+a_{\min }\right) \\
\text { with amax and amin the maximum and minimum flow acceleration }\end{array}$ \\
\hline ctr & corrected trochoidal wave \\
\hline sos & second-order Stokes \\
\hline unet & net current velocity at reference level zref \\
\hline$Z_{\text {ref }}$ & reference level \\
\hline
\end{tabular}

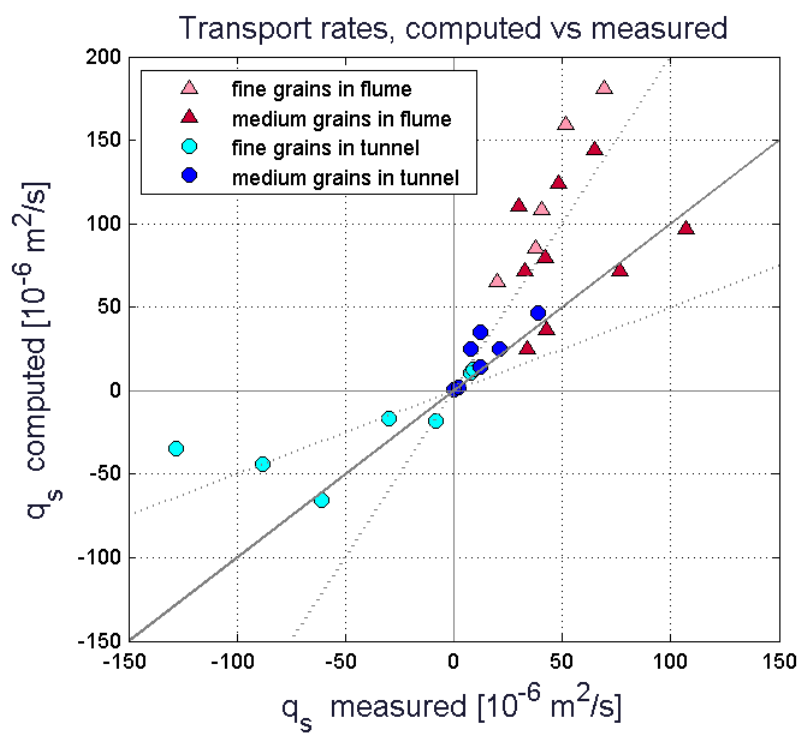

Figure 3. Computed against measured sediment transport rates $q_{s}$ for medium and fine $\left(d_{50}<0.15 \mathrm{~mm}\right)$ sediments in flume and tunnel. Conditions according to Table 1.

Figure 3 shows measured and computed transport rates of fine and medium grain sizes in flume and tunnel for the conditions of Table 1. Circles and triangles denote tunnel and flume conditions respectively. Within these categories we distinguish between medium and fine grain sizes. Figure 3 shows that the trends in the transport rates measured in flume and tunnel are fairly well reproduced by the model: both the model and the measurements show positive transport for fine sediment in a flume under waves with strong velocity skewness, while the tunnel conditions that resulted for fine grains in negative transport also lead to negative transport in the model.

\section{Flume and tunnel simulations with equal velocity skewness}

The absence of negative transport of fine sediment under real waves is an important result that already gives an indication of the important effect of the hydrodynamic differences between flume and tunnel on sediment transport. But for a fair study of its influence on transport rates, it is important to exclude the influence of differences in wave shape. This is especially relevant for fine grains, since its transport has been shown to be highly dependent on the degree of skewness (Dohmen-Janssen et al., 2002b; Hassan and Ribberink, 2010). Therefore we define a number of tunnel conditions with increasing energy but constant velocity skewness $R$ and use linear wave theory to determine linearly a matching surface elevation signal that can be used as input for the flume simulations. In this way we can simulate tunnel and flume situations with a similar oscillating velocity signal at the edge of the 
boundary layer. Subsequently, we compute transport rates under the defined tunnel and flume conditions for two different grain sizes. An overview of the conditions is given in Table 2.

\begin{tabular}{|c|c|c|c|c|c|c|c|c|c|}
\hline \multirow[b]{2}{*}{ code } & \multirow[b]{2}{*}{$\begin{array}{c}\mathrm{T} \\
(\mathrm{s}) \\
\end{array}$} & \multirow[b]{2}{*}{$\begin{array}{r}\mathrm{d} 50 \\
(\mathrm{~mm}) \\
\end{array}$} & \multirow[b]{2}{*}{$\mathrm{R}$} & \multirow[b]{2}{*}{ B } & \multirow[b]{2}{*}{$\begin{array}{l}\text { Wave } \\
\text { shape }\end{array}$} & \multicolumn{2}{|c|}{ tunnel } & \multicolumn{2}{|c|}{ flume $(h=3.5 \mathrm{~m})$} \\
\hline & & & & & & $\begin{array}{c}\mathrm{u} 1 \\
(\mathrm{~m} / \mathrm{s}) \\
\end{array}$ & $\begin{array}{c}\mathrm{u} 2 \\
(\mathrm{~m} / \mathrm{s}) \\
\end{array}$ & $\begin{array}{l}\mathrm{a} 1 \\
(\mathrm{~m}) \\
\end{array}$ & $\begin{array}{l}\text { a2 } \\
(\mathrm{m}) \\
\end{array}$ \\
\hline R62f01 & 6.5 & 0.138 & 0.620 & 0.500 & sos & 0.500 & 0.120 & 0.336 & 0.096 \\
\hline R62f02 & 6.5 & 0.138 & 0.620 & 0.500 & sos & 0.600 & 0.144 & 0.404 & 0.116 \\
\hline R62f03 & 6.5 & 0.138 & 0.620 & 0.500 & sos & 0.690 & 0.166 & 0.464 & 0.133 \\
\hline R62f04 & 6.5 & 0.138 & 0.620 & 0.500 & sos & 0.780 & 0.187 & 0.525 & 0.150 \\
\hline R62f05 & 6.5 & 0.138 & 0.620 & 0.500 & sos & 0.860 & 0.206 & 0.579 & 0.166 \\
\hline R62f06 & 6.5 & 0.138 & 0.620 & 0.500 & sos & 0.940 & 0.226 & 0.632 & 0.181 \\
\hline R62f07 & 6.5 & 0.138 & 0.620 & 0.500 & sos & 1.010 & 0.242 & 0.679 & 0.195 \\
\hline R62f08 & 6.5 & 0.138 & 0.620 & 0.500 & sos & 1.080 & 0.259 & 0.727 & 0.208 \\
\hline R62f09 & 6.5 & 0.138 & 0.620 & 0.500 & sos & 1.140 & 0.274 & 0.767 & 0.220 \\
\hline$R 62 f 10$ & 6.5 & 0.138 & 0.620 & 0.500 & sos & 1.200 & 0.288 & 0.807 & 0.231 \\
\hline $\mathrm{R} 62 \mathrm{f} 11$ & 6.5 & 0.138 & 0.620 & 0.500 & sos & 1.250 & 0.300 & 0.841 & 0.241 \\
\hline $\mathrm{R} 62 \mathrm{~m} 01$ & 6.5 & 0.245 & 0.620 & 0.500 & sos & 0.500 & 0.120 & 0.336 & 0.096 \\
\hline $\mathrm{R} 62 \mathrm{~m} 02$ & 6.5 & 0.245 & 0.620 & 0.500 & sos & 0.600 & 0.144 & 0.404 & 0.116 \\
\hline $\mathrm{R} 62 \mathrm{~m} 03$ & 6.5 & 0.245 & 0.620 & 0.500 & sos & 0.690 & 0.166 & 0.464 & 0.133 \\
\hline $\mathrm{R} 62 \mathrm{~m} 04$ & 6.5 & 0.245 & 0.620 & 0.500 & sos & 0.780 & 0.187 & 0.525 & 0.150 \\
\hline $\mathrm{R} 62 \mathrm{~m} 05$ & 6.5 & 0.245 & 0.620 & 0.500 & sos & 0.860 & 0.206 & 0.579 & 0.166 \\
\hline $\mathrm{R} 62 \mathrm{~m} 06$ & 6.5 & 0.245 & 0.620 & 0.500 & sos & 0.940 & 0.226 & 0.632 & 0.181 \\
\hline $\mathrm{R} 62 \mathrm{~m} 07$ & 6.5 & 0.245 & 0.620 & 0.500 & sos & 1.010 & 0.242 & 0.679 & 0.195 \\
\hline $\mathrm{R} 62 \mathrm{~m} 08$ & 6.5 & 0.245 & 0.620 & 0.500 & sos & 1.080 & 0.259 & 0.727 & 0.208 \\
\hline $\mathrm{R} 62 \mathrm{~m} 09$ & 6.5 & 0.245 & 0.620 & 0.500 & sos & 1.140 & 0.274 & 0.767 & 0.220 \\
\hline $\mathrm{R} 62 \mathrm{~m} 10$ & 6.5 & 0.245 & 0.620 & 0.500 & sos & 1.200 & 0.288 & 0.807 & 0.231 \\
\hline $\mathrm{R} 62 \mathrm{~m} 11$ & 6.5 & 0.245 & 0.620 & 0.500 & sos & 1.250 & 0.300 & 0.841 & 0.241 \\
\hline
\end{tabular}

With:

$\mathrm{u} 1, \mathrm{u} 2$ amplitude of $1^{\text {st }} / 2^{\text {nd }}$ harmonic of the horizontal velocity (input tunnel simulation)

a1, a2 amplitude of $1^{\text {st }} / 2^{\text {nd }}$ harmonic of the surface elevation (input flume simulation)

Figure 4 shows computed transport rates of fine and medium grain sizes in flume and tunnel for the conditions of Table 2. Both for fine and medium grain sizes the flume simulations show a shift of the transport rates in onshore direction compared to the tunnel situation. This shift is the largest for fine sediment and can, in that case, even cover a shift from offshore to onshore transport.

As explained in the introduction and described by Dohmen-Janssen et al. (2002), the slow settlement of fine grains can lead to a phase lag between velocity and concentration, high amounts of sediment in suspension at the moment of flow reversal and, in case of velocity-skewed waves, to offshore transport rates. This effect increases with increasing wave energy, preserving the shape of the velocity signal. The present simulations show that this effect is completely overruled by the hydrodynamic differences between flume and tunnel. Not only induce the differences a shift of the transport rates in onshore direction, but this shift also depends on the grain size. To investigate whether we can attribute this shift completely to the additional net current in the flume, we carry out a number of model experiments in the next section. 

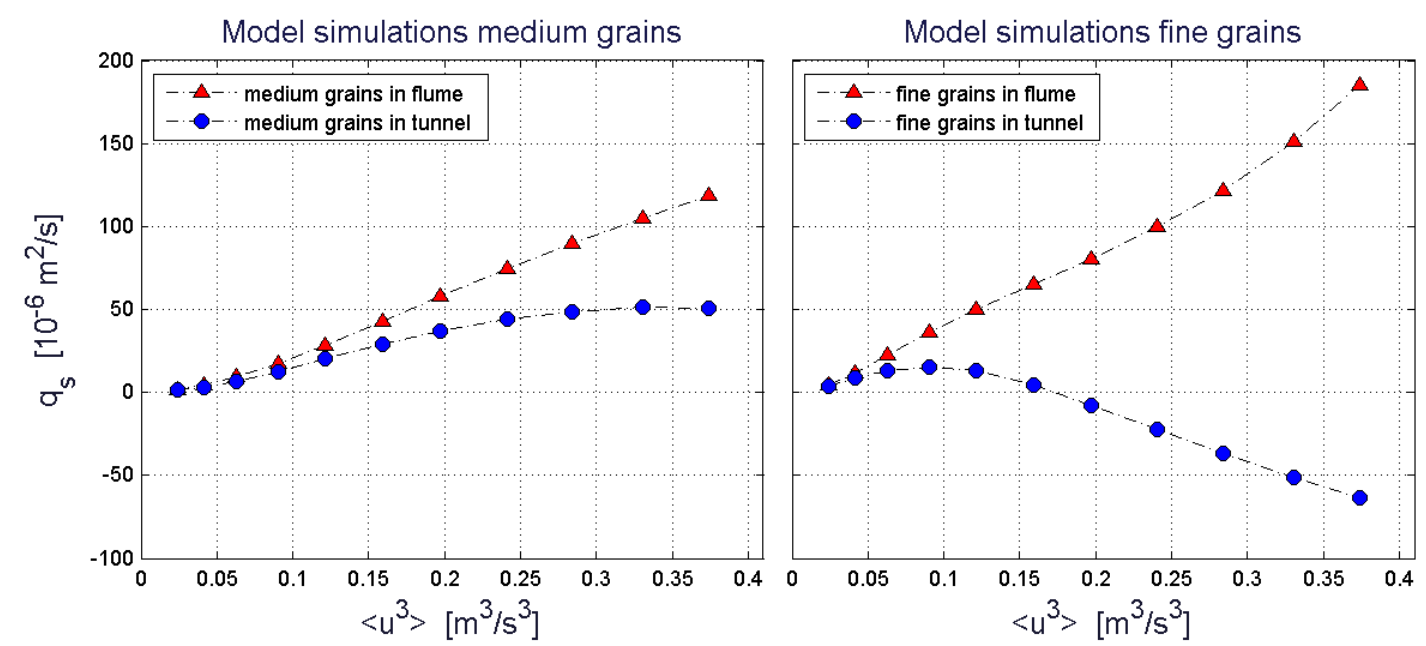

Figure 4. Computed sediment transport rates $q_{\mathrm{s}}$ as function of $\left\langle u^{3}\right\rangle$ for medium $(0.245 \mathrm{~mm})$ and fine $(0.138 \mathrm{~mm})$ sediments in flume and tunnel. Conditions according to Table 2 . Note that $\left\langle u^{3}\right\rangle$ has been computed from the reduced velocity time series $\underline{u}_{\mathrm{red}}=u(\mathrm{t})-U$ in the tunnel at $100 \mathrm{~mm}$ above the bed level.

\section{MODEL EXPERIMENTS}

\section{The role of real wave induced streaming}

The turbulence in the wave boundary layer causes a shift of the phase of the horizontal orbital velocity over the vertical. In a wave flume, where horizontal gradients and vertical orbital velocities occur, this phase shift induces a net downward transport of positive horizontal momentum (LonguetHiggins, 1958). This momentum transport results in a net onshore current or 'real wave induced streaming', which might explain the different sediment transport rates for flume and tunnel. An additional net onshore current, i.e. a shift of the velocity signal in positive direction, could contribute to increased onshore transport in various ways: Firstly, the onshore directed transport will last longer and take place with higher velocities, while the duration of the offshore directed transport will be shortened and the velocity will be reduced. Secondly, increased shear and increased turbulence intensities in the onshore phase of the horizontal velocity will lead to increased pick up of sediment, subsequently stirred up to higher levels.

To investigate whether the real wave induced streaming can be the full explanation of the increased transport rates in the flume, we compare the flume simulations of the previous section with simulations in which the current generating mechanism has been turned off. When we just turn of the wave-current interaction term $F_{\text {wci }}$ in the wave-averaged momentum equation not only the real wave induced onshore streaming, but also the offshore streaming generated by asymmetry in turbulence is excluded from the simulation. This would imply an unfair comparison, because the latter is present both in flumes and tunnels. To exclude only the Longuet-Higgins streaming, we remove the vertical transport of horizontal momentum $(\stackrel{x}{w} \partial \stackrel{0}{u} / \partial z$ ) from the intra-wave horizontal momentum equation and thus exclude the $\bar{w} \frac{\alpha}{\partial u / \partial z}$-term from the determination of the wave-current interaction term. Note that this affects not only the wave-averaged current, but also slightly changes the orbital velocity signal. But, contrary to its contribution to the wave-averaged momentum, the intra-wave contribution of the $\stackrel{\alpha}{w} \partial \stackrel{u}{u} / \partial z$-term will be small compared to the other terms.

The flume simulations without real wave streaming show strongly reduced onshore transport rates compared to the earlier flume simulations (Figure 5). But the transport rates still show a considerable onshore shift compared to the tunnel simulation. They also do not show the tendency of a decreasing growth with increasing energy, as was the case for tunnel simulations with fine grains. The phase-lag effect seems still to be suppressed or at least overruled by other differences between tunnel and flume. Therefore we conclude that the additional onshore current in the flume does contribute to onshore sediment transport, but can not be the full explanation of the differences in transport rates in tunnel and flume. 

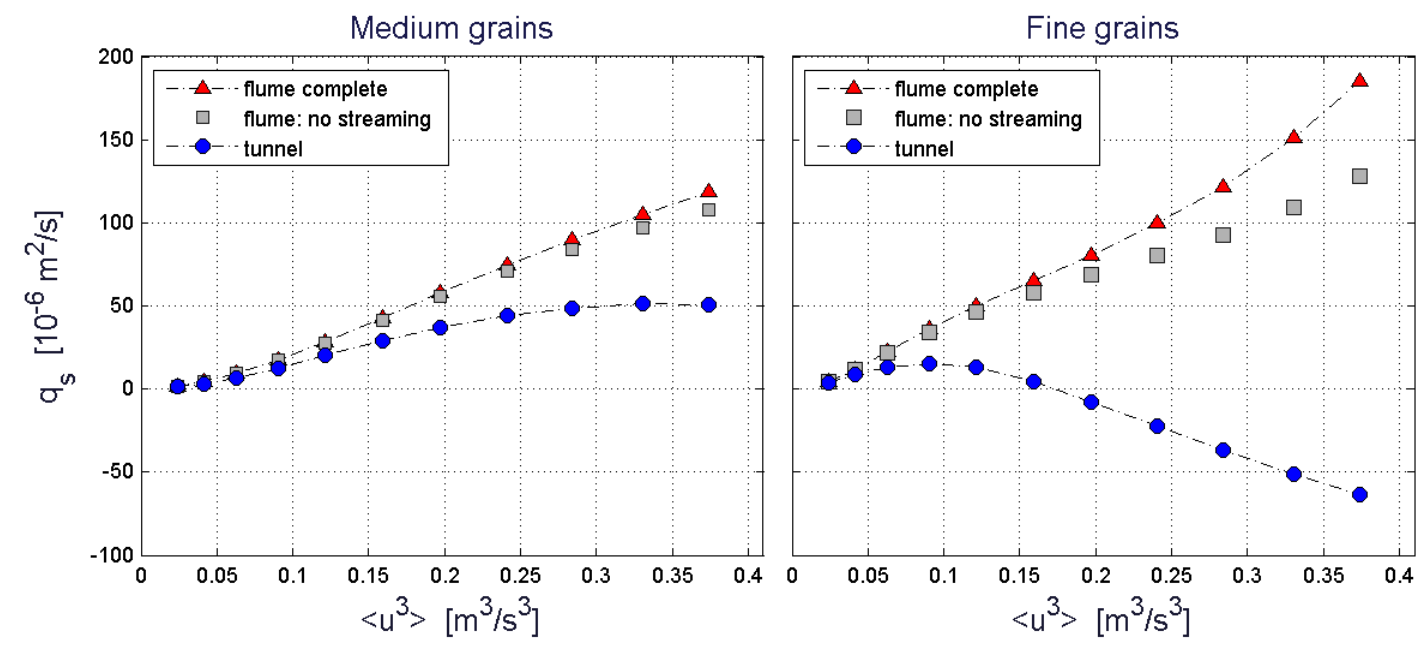

Figure 5. Computed sediment transport rates qs as function of $<u 3>$ for medium $(0.245 \mathrm{~mm})$ and fine $(0.138 \mathrm{~mm})$ sediments in flume and tunnel. Conditions according to Table 2. ADDED: flume without streaming.

\section{The role of vertical sediment advection}

The presence of vertical orbital velocities in the flume could contribute also to onshore transport via the vertical advection of sediment. The orbital motion introduces a difference between the onshore and offshore phase of the wave: After the maximum onshore horizontal orbital velocity the orbital motion will be downwards, while the motion will be upwards after maximum offshore velocity. This will influence the concentration profile when sediment is present at levels where the vertical velocity $\underset{w}{w}$ is in the order of the settling velocity of the grains $w_{s}$. The compression and stretching of the concentration profile causes the concentration at the higher levels to decrease faster after the onshore movement and slower after the offshore movement. This influences the phase-lag between velocity and concentration: at that level, less sediment is present at the on- to offshore flow reversal, so less sediment will be transported offshore, while the higher concentration at the off- to onshore flow reversal leads to more onshore transport.

Estimates of the fall velocities (using Van Rijn, 1993), stirring up levels (with sheet-flow layer thickness according to Wilson, 1989) and vertical orbital velocities for representative conditions show that this process will not be relevant for the medium grain size used before. For that size, the sediment will not be stirred up to levels where the vertical orbital velocity approaches the fall velocity. But this process might play a rol in the transport of the fine sediment.

To investigate the role of vertical sediment advection we switch on/off the vertical advection in the sediment balance:

$\frac{\partial c}{\partial t}+\frac{\partial(U+\stackrel{o}{u}) c}{\partial x}+\frac{\partial\left(\stackrel{\infty}{w}+w_{s}\right) c}{\partial z}=\frac{\partial}{\partial z}\left(\varepsilon \frac{\partial c}{\partial z}\right) \Leftrightarrow \frac{\partial c}{\partial t}+\frac{\partial(U+\stackrel{o}{u}) c}{\partial x}+\frac{\partial w_{s} c}{\partial z}=\frac{\partial}{\partial z}\left(\varepsilon \frac{\partial c}{\partial z}\right)$

Figure 6 shows a comparison between a simulation with and without vertical sediment advection. These simulations are carried out with a sinusoidal surface elevation and the onshore streaming mechanism has been turned off. Time series and profiles of the concentration illustrate the working of the mechanism. The sediment transport rates for the conditions of Table 2 are shown in Figure 8 (red pentagram). We conclude from this figure that the vertical advection of sediment that is present in flumes but not in tunnels, is indeed able to influence the rate of sediment transport for fine grains, but that its contribution to the onshore transport is small compared the contribution of the real wave induced streaming. 

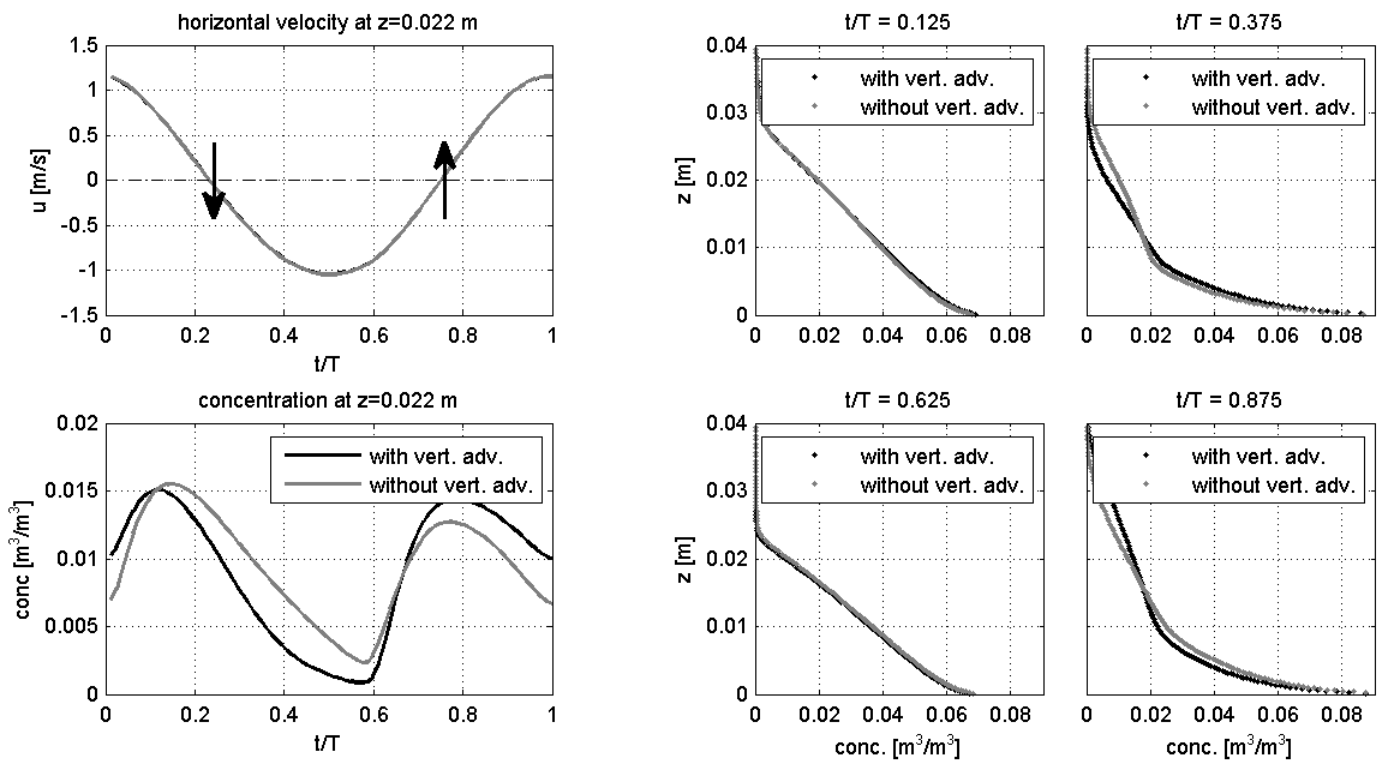

Figure 6. Sediment concentrations computed with and without advection of sediment by the vertical orbital motion. Conditions: sinusoidal surface wave $T=6.5 \mathrm{~s}, H=1.25 \mathrm{~m}, h=3.5 \mathrm{~m}, d_{50}=1 \mathrm{E}-4 \mathrm{~m}$.

\section{The role of horizontal sediment advection}

A third mechanism that contributes to increased onshore sediment transport in flumes is related to the (intra-wave) horizontal gradients under surface waves. A direct effect of the horizontal gradient in the horizontal orbital velocity $\tilde{u}$ is reduction of the distance between particles in front of the wave top and augmentation behind the wave top. This leads to an amplification of the sediment concentration peak at maximum velocity in the direction of propagation, while the peak during the offshore phase of the wave will be reduced. Concurrence of a larger peak in concentration with maximum onshore velocities and of a reduced concentration with maximum offshore velocities will contribute to onshore directed sediment transport. This mechanism would even cause net onshore transport for a purely sinusoidal wave in the absence of the net current.

A simple analytical illustration of this process is given in appendix A, neglecting vertical diffusive and advective sediment exchange. This description demonstrates the influence of the ratio of orbital velocity and propagation speed, i.e. $\tilde{u} / c_{\mathrm{p}}$, which forms actually an indicator of non-linearity.

Note also the parallel with the generation of Stokes drift, where Lagrangian description of the motion of a water particle shows the gradual onshore displacement and the elongated duration of onshore movement. To investigate the contribution of the gradients in advection in the Eulerian framework of the present model we switch on/off the term of the sediment balance that denotes the advection by horizontal orbital velocities:

$$
\frac{\partial c}{\partial t}+\frac{\partial(U+\stackrel{o}{u}) c}{\partial x}+\frac{\partial w_{s} c}{\partial z}=\frac{\partial}{\partial z}\left(\varepsilon \frac{\partial c}{\partial z}\right) \Leftrightarrow \frac{\partial c}{\partial t}+\frac{\partial U c}{\partial x}+\frac{\partial w_{s} c}{\partial z}=\frac{\partial}{\partial z}\left(\varepsilon \frac{\partial c}{\partial z}\right)
$$

Figure 7 shows a comparison between simulations with and without (orbital) horizontal sediment advection. These simulations are carried out with a sinusoidal surface elevation and small grains $\left(d_{50}=\right.$ $0.1 \mathrm{~mm}$ ). In all simulations onshore streaming and vertical sediment advection have been turned off. We use again time series and profiles of the concentration to study the mechanism. The concentrations obtained with horizontal sediment advection exceed the concentrations obtained without advection almost during the complete onshore movement and over the entire profile. During the offshore movement, the situation is opposite. Simulations with fine and medium grain sizes show similar behaviour. So, contrary to vertical sediment advection, we may expect the gradients in the horizontal sediment advection to contribute to onshore transport both for fine and medium grain sizes. 

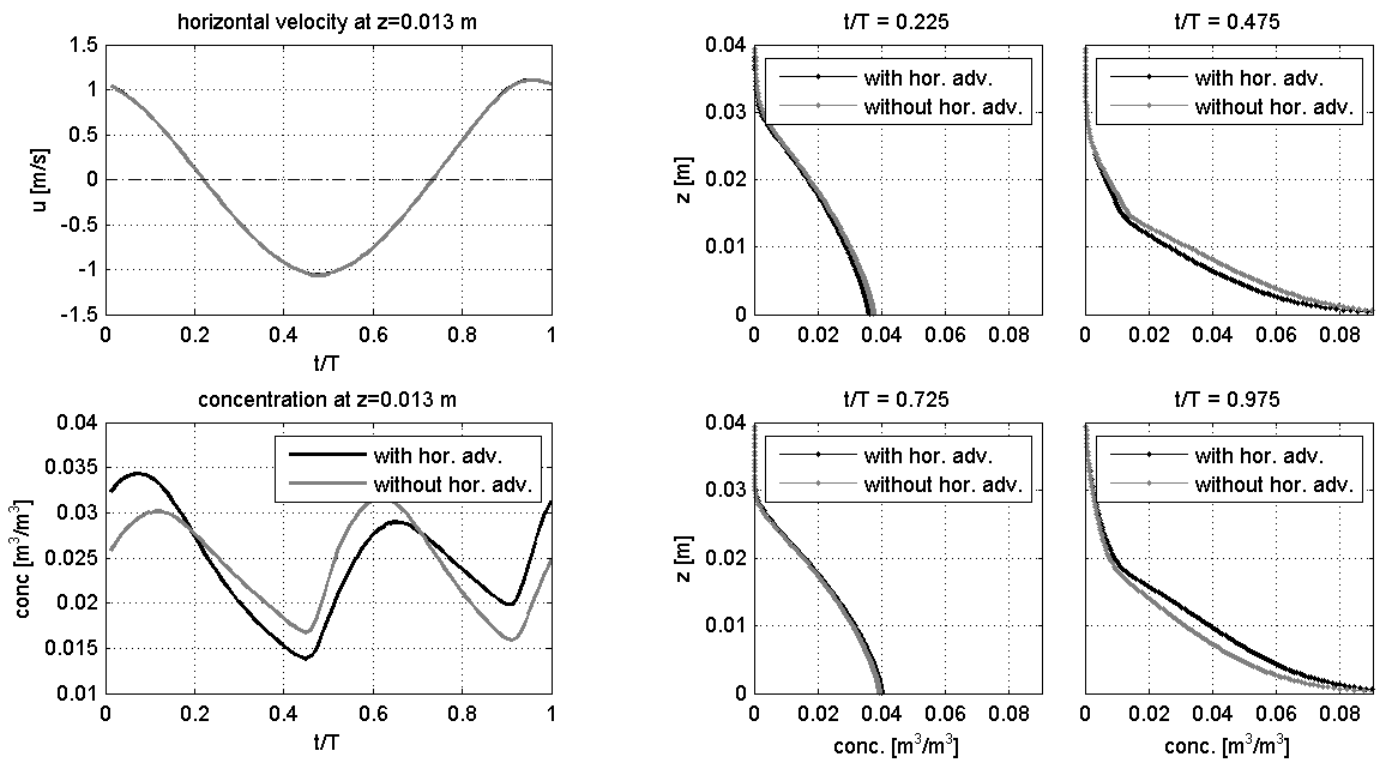

Figure 7. Sediment concentrations computed with and without advection of sediment by the horizontal orbital motion. Conditions: sinusoidal surface wave $T=6.5 \mathrm{~s}, H=1.25 \mathrm{~m}, h=3.5 \mathrm{~m}, d_{50}=1 \mathrm{E}-4 \mathrm{~m}$.
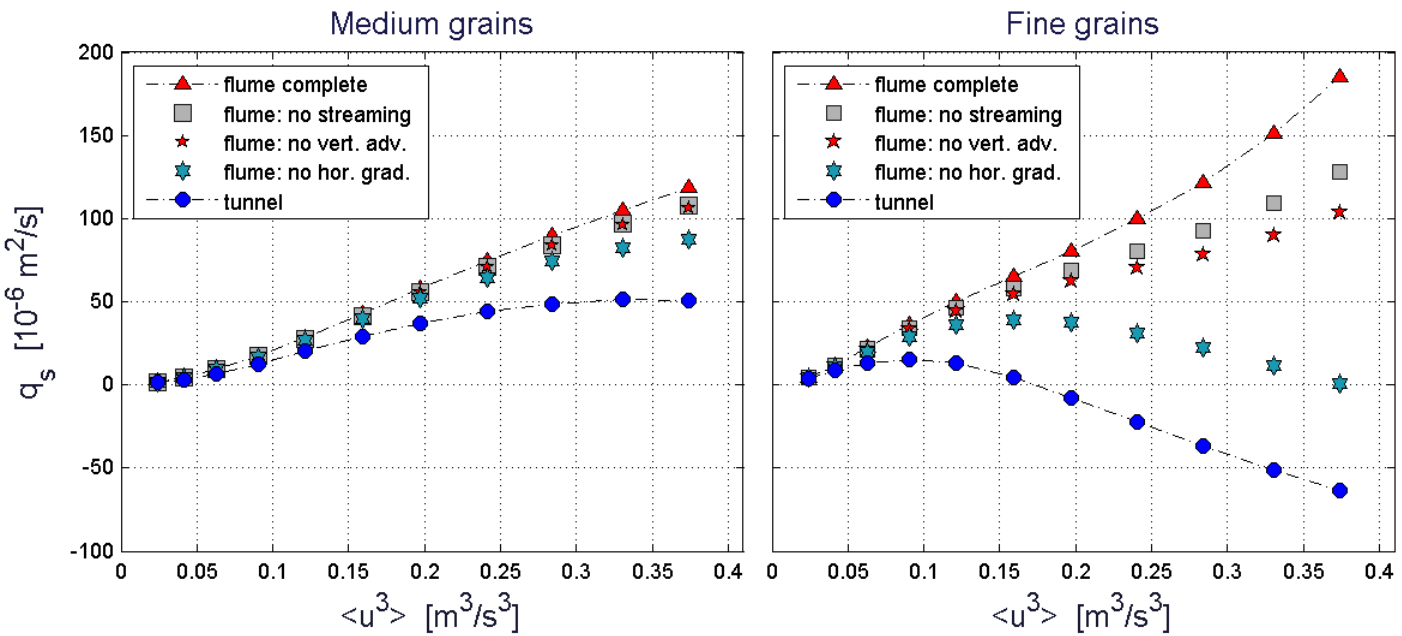

Figure 8. Computed sediment transport rates $q_{\mathrm{s}}$ as function of $\left\langle u^{3}\right\rangle$ for medium $(0.245 \mathrm{~mm})$ and fine $(0.138 \mathrm{~mm})$ sediments in flume and tunnel. Conditions according to Table 2. ADDED: flume simulations with advection of sediment by the vertical orbital motion turned off (red pentagram) and flume simulations with advection of sediment by the horizontal orbital motion turned off (light blue hexagram).

Sediment transport rates for the conditions of table 2 are shown in Figure 8. We conclude from this figure that the real wave induced intra-wave gradients in horizontal sediment flux contribute to increased onshore transport rates in flumes both for fine and medium grain sizes and that its contribution is of equal importance as the contribution of the real wave induced streaming that was initially expected to be the most important difference between tunnels and waves.

\section{SUMMARY AND CONCLUSIONS}

The influence of the hydrodynamic differences between oscillating flow tunnels and wave flumes on sediment transport under waves in the sheet flow regime has been investigated by numerical simulations. The main question was whether the additional net onshore current that will be present under real waves in a flume (Longuet-Higgins streaming) can explain the differences between measured sediment transport rates in tunnel and flume experiments. We investigate this question with a 
1DV RANS model that can simulate both a wave flume and an oscillating flow tunnel. The flumeversion explicitely simulates the generation of a net current by the waves in a closed flume, including the Longuet-Higgins streaming. Considering the hypothesis, special attention has been given to the validation of the hydrodynamics of the flume model on the net current profile. The main results are:

- Model predictions of the wave-generated net current show very good agreement with the net current profiles as measured by Klopman (1994) in his small scale fixed bed flume measurements. Also the predicted horizontal orbital velocities match well with the measurements. This forms a validation of the hydrodynamic formulations of the model.

- The hydrodynamic differences between wave flume and oscillating flow tunnel do influence the transport of sediments. This is especially true for fine sediment. In agreement with the recent flume measurements of Schretlen, the present flume simulations show a shift of the transport rates in onshore direction compared to the tunnel situation. This shift can even cover a shift from offshore to onshore transport for fine sediment under $2^{\text {nd }}$ order Stokes waves in a tunnel, respectively a flume.

- The real wave induced onshore streaming indeed contributes to sediment transport in onshore direction, but can not be the only explanation for increased onshore transport rates in flumes.

- For fine grains, the presence of vertical orbital velocities in wave flumes does also contribute to increased onshore transport rates in flumes by vertical advection of sediment. At the end of the onshore/offshore phase of the horizontal orbital velocity, the vertical orbital motion is downward/upward, which causes the sediment to settle faster/slower. The effect is a decrease of transport during the offshore phase and an increase of transport during the onshore phase of the wave.

- The presence of horizontal gradients in the horizontal sediment flux in wave flumes does also contribute to increased onshore transport rates in flumes. The gradients in the flux cause an amplified peak in concentration at maximum onshore velocity and a reduced concentration at maximum offshore velocity. The concurrence of large onshore velocities and large concentrations leads to increased onshore transport. This mechanism itself is independent of grain sizes, but the actual contribution to transport rates depends on the amount of sediment in suspension, which is of course dependent on the grain size. In the investigated wave conditions, this process is of equal importance for the transport of sediment as the wave-induced streaming and should therefore be considered in the (formulae used in) morphodynamic modeling.

Comparison of model results with measured sediment concentrations and systematic exploration of the parameter space using the model are expected to give additional insight in the working of the various real wave effects discussed above and their relative contribution to onshore sediment transport and will be the focus of future work.

\section{ACKNOWLEDGMENTS}

The first author wishes to thank Jolanthe Schretlen for putting at his disposal the partly still unpublished data obtained during her 2007 \& 2008 wave flume experiments and for giving him the opportunity to join the 2008 measurement campaign in the Large Wave Flume (GWK) in Hannover.

This study would not have been possible without the Integrated Infrastructure Initiative HydralabIII-SANDS of the European Community's Sixth Framework Programme and the SANTOSS project. The latter is a collaboration project between Twente University (The Netherlands) and Aberdeen University (UK), with input from Liverpool University (UK), Bangor University (UK) and DELTARES (The Netherlands), funded by the Dutch Technology Foundation STW and the UK's Engineering and Physical Sciences Research Council (EPSRC).

\section{APPENDIX A}

The contribution of intra-wave gradients in horizontal advection to sediment transport in the propagation direction of the wave can be analytically illustrated as follows:

Moving with the wave propagation speed $c_{\mathrm{p}}$, the material derivative of a steady harmonic oscillation is zero for all quantities, including the sediment flux $f=\tilde{u} c$ : 


$$
\frac{\partial f}{\partial t}+c_{p} \frac{\partial f}{\partial x}=0
$$

We substitute this equality into the sediment balance, neglecting all vertical sediment exchange:

$$
\frac{\partial c}{\partial t}+\frac{\partial \stackrel{o}{u} c}{\partial x}=\frac{\partial c}{\partial t}-\frac{1}{c_{p}} \frac{\partial \stackrel{o}{u} c}{\partial t}=\frac{\partial}{\partial t}\left\{c-\frac{\stackrel{\circ}{u} c}{c_{p}}\right\}=0
$$

An expression for sediment concentration $c$ derived from this equation shows the variation of $c$ with $\tilde{u}$ ( $\alpha$ is constant). Taylor expansion around $\tilde{u} / c_{\mathrm{p}} \approx 0$ yields an approximation valid for $\tilde{u} / c_{\mathrm{p}}<<1$.

$$
c(x, t)=\alpha\left(1-\frac{\stackrel{\circ}{u}(x, t)}{c_{p}}\right)^{-1} \approx \alpha\left(1+\frac{\stackrel{\circ}{u}(x, t)}{c_{p}}\right)
$$

Multiplication with $\tilde{u}$ gives an expression for the flux $f$ that shows the period averaged contribution to onshore sediment transport:

$$
f(x, t) \approx \alpha \% \frac{\alpha u^{2}}{c_{p}} \rightarrow \bar{f} \approx \frac{\alpha d^{2}}{2 c_{p}}
$$

\section{REFERENCES}

Bosboom. J. and Klopman. G. 2000. 1DV simulation of wave current interaction. Proc. 27th ICCE, ASCE, 2452-2466.

Davies, A.G. and Li, Z. 1997. Modelling sediment transport beneath regular symmetrical and asymmetrical waves above a plane bed. Continental Shelf Research, 17(5), 555-582.

Dohmen-Janssen, C.M., 1999. Grain size influence on sediment transport in oscillatory sheet flow phase lags and mobile-bed effects, PhD-Thesis, Delft University of Technology, Delft, 246 pp.

Dohmen-Janssen, C.M. and Hanes, D.M., 2002. Sheet flow dynamics under monochromatic nonbreaking waves. Journal of Geophysical Research, 107(C10)

Dohmen-Janssen, C.M., Kroekenstoel, D.F., Hassan, W.N., Ribberink, J.S., 2002. Phase lags in oscillatory sheet flow: experiments and bed load modelling. Coastal Enigneering, 46, 61-87

Dohmen-Janssen, C.M., and Hanes, D.M., 2005, Sheet flow and suspended sediment due to wave groups in a large wave flume, Continental Shelf Research, 25, 333-347

Elgar, S., and R. T. Guza (1985), Observations of bispectra of shoaling surface gravity waves, Journal of Fluid Mechanics, 161, 425- 448.

Hassan, W.N. and Ribberink J.S., 2010, Modelling of sand transport under wave-generated sheet flows with a RANS diffusion model; Coastal Engineering, 57, 19-29

Henderson, S.M., Allen, J.S., Newberger, P.A., 2004. Nearshore sandbar migration predicted by an eddy-diffusive boundary layer model. Journal of Geophysical Research, 109(C06024).

Hoefel, F. and Elgar, S., 2003. Wave-induced sediment transport and sandbar migration. Science, 299, 1885-1887.

Holmedal, L.E. and Myrhaug, D., 2009, Wave-induced steady streaming, mass transport and net sediment transport in rough turbulent ocean bottom boundary layers, Continental Shelf Research, 29.7, 911-926

Klopman, G. 1994. Vertical structure of the flow due to waves and currents. Progress report, Delft Hydraulics, H840.32, Part 2.

Longuet-Higgins, M.S., 1953, Mass transport in water waves, Philos. Trans. R. Soc. London, Ser. A, $245,535-581$.

Longuet-Higgins, M.S., 1958, The mechanics of the boundary-layer near the bottom in a progressive wave". Proc. 6th ICCE, ASCE, 184-193.

O'Donoghue, T. and Wright, S., 2004a. Concentrations in oscillatory sheet flow for well sorted and graded sands. Coastal Engineering, 50: 117 - 138.

O'Donoghue, T. and Wright, S., 2004b. Flow tunnel measurements of velocities and sand flux in oscillatory sheet flow for well-sorted and graded sands. Coastal Engineering, 51, 1163 - 1184. 
Ribberink, J.S. and Al-Salem, A.A., 1994. Sediment transport in oscillatory boundary layers in cases of rippled beds and sheet flow. Journal of Geophysical Research, 99(C6), 12707 - 12727.

Ribberink, J.S. and Al-Salem, A.A., 1995. Sheet flow and suspension of sand in oscillatory boundary layers. Coastal Engineering, 25, 205 - 225.

Ribberink, J.S. and Chen, Z., 1993. Sediment transport of fine sand under asymmetric oscillatory flow. Experimental study in the large oscillating water tunnel of Delft Hydraulics, Delft University of Technology, Delft Hydraulics, Delft, The Netherlands.

Richardson, J.F. \& Zaki, W.N. 1954 Sedimentation and fluidization. Trans. Inst. Chem. Engng, 32, 35-53.

Rijn, L.C. van, 1993. Principles of sediment transport in rivers, estruaries and coastal seas. Aqua Publ. (The Netherlands)

Ruessink, B.G., van den Berg, T.J.J., van Rijn, L.C., 2009. Modeling sediment transport beneath skewed-asymmetric waves above a plane bed. Journal of Geophysical Research, 114(C11021)

Schretlen, J.L.M., Ribberink J.S.,O’Donoghue, T., 2010. Boundary layer flow and sand transport under full scale surface waves, Proc. 32th ICCE, ASCE

Trowbridge, J. and Madsen, O. S., 1984, Turbulent wave boundary layers. 2. Second-order theory and mass transport. Journal of Geophysical Research, 89(C5), 7999-8007.

Uittenbogaard, R.E., 2000. 1DV simulation of wave current interaction. Proc. 27th ICCE, ASCE, 255268

Werf, J. van der, Schretlen, J., Ribberink, J., O'Donoghue, T., 2009. Database of full-scale laboratory experiments on wave-driven sand transport processes. Coastal Engineering., 56, 726-732

Wilson, K.C., 1989, Friction of wave-induced sheet flow, Coastal Engineering, 12, 371-379.

Wright, S. and O'Donoghue, T., 2002. Total sediment transport rate predictions in wave current sheet flow with graded sand. Oscillatory flow tunnel experiments at Aberdeen University, University of Aberdeen, Aberdeen, UK.

Zyserman, J.A. and Fredsoe, J., 1994. Data analysis of bed concentration of suspended sediment. Proc. Am. Soc. Civ. Eng., Journal of Hydraulic Engineering, 120(9): 1021-1042. 